Article

\title{
An Elderly Health Monitoring System Using Machine Learning and In-Depth Analysis Techniques on the NIH Stroke Scale
}

\author{
Jaehak Yu ${ }^{1}$, Sejin Park ${ }^{2}$, Hansung Lee ${ }^{3}$,, Cheol-Sig Pyo ${ }^{1}$ and Yang Sun Lee ${ }^{4, *(\mathbb{D})}$ \\ 1 Department of KSB (Knowledge-converged Super Brain) Convergence Research, ETRI (Electronics and \\ Telecommunications Research Institute), Daejeon 34129, Korea; dbzzang@etri.re.kr (J.Y.); \\ cspyo@etri.re.kr (C.-S.P.) \\ 2 Research Team for Health \& Safety Convergence, Korea Research Institute of Standards and Science (KRISS), \\ Daejeon 34113, Korea; sjpark@kriss.re.kr \\ 3 Computer Engineering, Youngsan University, Kyungnam-do 50510, Korea; mohan@ysu.ac.kr \\ 4 Division of Convergence Computer and Media, Mokwon University, Daejeon 35349, Korea \\ * Correspondence: yslee48@gmail.com; Tel.: +82-42-829-7638
}

Received: 5 June 2020; Accepted: 2 July 2020; Published: 6 July 2020

\begin{abstract}
Recently, with the rapid change to an aging society and the increased interest in healthcare, disease prediction and management through various healthcare devices and services is attracting much attention. In particular, stroke, represented by cerebrovascular disease, is a very dangerous disease, in which death or mental and physical aftereffects are very large in adults and the elderly. The sequelae of such stroke diseases are very dangerous, because they make social and economic activities difficult. In this paper, we propose a new system to prediction and in-depth analysis stroke severity of elderly over 65 years based on the National Institutes of Health Stroke Scale (NIHSS). In addition, we use the algorithm of decision tree of C4.5, which is a methodology of prediction and analysis of machine learning techniques. The $\mathrm{C} 4.5$ decision trees are machine learning algorithms that provide additional in-depth rules of the execution mechanism and semantic interpretation analysis. Finally, in this paper, it is verified that the C 4.5 decision tree algorithm can be used to classify and predict stroke severity, and to obtain additional NIHSS features reduction effects. Therefore, during the operation of an actual system, the proposed model uses only 13 features out of the 18 stroke scale features, including age, so that it can provide faster and more accurate service support. Experimental results show that the system enables this by reducing the patient NIH stroke scale measurement time and making the operation more efficient, with an overall accuracy, using the C4.5 decision tree algorithm, of $91.11 \%$.
\end{abstract}

Keywords: National Institutes of Health Stroke Scale (NIHSS); health monitoring system; stroke analysis; machine learning; stroke severity prediction

\section{Introduction}

The number of deaths in Korea in 2015 was recently reported to be 275,895 [1]. The cause of death was reported as malignant neoplasm $(76,855)$, heart disease $(28,326)$, and cerebrovascular disease $(24,455)$ [1]. In particular, the death rate from cerebrovascular disease in Koreans is 48 per 100,000 people, followed by malignant neoplasm and heart disease. The number of deaths due to cerebrovascular disease in Korea has been declining since 2005, but it is the second leading cause of death due to a single disease after malignant neoplasm [1-3]. According to the analysis information of these institutions, Korea is continuously growing in chronic diseases due to aging. In particular, according to a recent study, the elderly over 60 years of age are showing an increasing mortality rate 
from the cardiovascular system disease [3-6]. The stroke is difficult to reliably evaluate the stroke and neurological damage, due to the various symptoms and the location of the stroke. In particular, the early detection of stroke is important for a person without a past medical history, but the probability of recurrence is nine times higher for people with a past history of stroke. As a result, in order to diagnose and treat physicians within a short period of time, research should be conducted for the continuous monitoring and management of patients with stroke.

According to recent studies, National Institutes of Health Stroke Scale (NIHSS)-based research topic objectively validates stroke severity and various studies are under way to stroke detection and use risk factors to prevent recurrence [7-11]. Since the Mathew scale was first published in 1972, methods for evaluating a stroke patient's disorder include the European stroke scale, the Scandinavian stroke scale, and the NIHSS of the American National Institutes of Health [12,13]. Among the various NIHSS methodologies, the method proposed by Bortt et al. is widely used tool as measurement criteria after stroke [7-9]. NIHSS measures 14 features associated with stroke severity, and statistically, it takes $6.6 \mathrm{~min}$ per patient. In addition, it is used as an evaluation health stroke scale, which is relatively easy to measure at the initial stage of a patient, and can be easily performed. According to various studies, stroke predictions can be quickly determined without imaging by using a scoring system based on the NIHSS that can accurately predict cortical damage in an acute ischemic stroke [14,15]. First, the severity of stroke in hospitalization for patients with ischemic stroke and the association of acute patients can be assessed. As such, the NIHSS is widely used globally as a tool, and it has already been verified through various studies for the reliability and validity of not only the examiner but also the test and retest. However, the NIHSS method is a tool to assess the extent of brain damage in stroke patients, but it has disadvantages, in that it does not provide an analytical result for the diagnosis of early stroke.

Another method of analyzing the risk factor of stroke is to find risk factors through previous research, health screening data, and clinical trials. Risk factors of stroke found in these studies include smoking, diabetes, obesity, and smoking [16-20]. Research has reported that stroke-related is not a single factor, but comes from the interaction of various and complex risk factors. Therefore, a new methodology for evaluating each stroke risk factor and deal with diseases early stroke detection or prediction. It is not known exactly when and where stroke disease occurs. Additionally, the recurrence rate of stroke varies according to the type, race, and risk factors, but clinical studies have reported that the recurrence rate is generally $10 \sim 15 \%$ within 1 year. Studies are underway to analyze stroke risk factors using various machine learning and statistical methodologies. Additionally, the proportional hazards model [20] proposed by Cox [21] or Weibull et al. [22] as a representative method, and Kannel et al. [23] have been reported, based on a logistic model. However, previous studies based on these risk factors are not suitable for predicting the risk of stroke in the elderly. These prior studies have the disadvantage that the stroke severity analysis and prediction system have a black box form, which is difficult to interpret and analyze automatically. Therefore, the provision of scientific analysis and interpretation information is essential, and should be provided as an analysis result. In particular, we need to find a new optimal stroke severity and analysis system for the elderly. Therefore, it is important to quickly detect and predict the early onset of stroke patients, but another important research issue is to find the recurrence of stroke in patients with a history of stroke.

In this paper, we propose a new system for predictive and in-depth analysis of stroke severity for the elderly over 65 years old, using NIHSS features and C4.5 decision tree algorithm. We propose a system that automatically classifies and analysis stroke severity into four classes using NIHSS features collected in real-time. The $\mathrm{C} 4.5$ decision tree are machine learning algorithms that provide additional in-depth rules of the execution mechanism and semantic interpretation analysis. Therefore, during the operation of an actual system, the proposed model uses only 13 features out of the 18 stroke scale features, including age, so that it can provide faster and more accurate service support. In this paper, it is verified that the $\mathrm{C} 4.5$ decision tree algorithm can be used to classify and predict stroke severity, and to obtain additional NIHSS features reduction effects. Finally, the system we developed in this 
study can be used to predict the risk of individual stroke recurrence, depending on the severity of the disorder and the type of risk factor by gender and by age group over 65 years.

The remainder of this paper is organized as follows. Section 2 discusses the main stroke diseases of the elderly and describes our research subjects and the machine learning for the stroke disease classification and prediction. Section 3 presents the proposed system of stroke severity prediction and in-depth analysis in the elderly. Section 4 describes the experimental results and analysis contents. In the Section 5, conclusions and future research are discussed.

\section{Materials and Methods}

\subsection{Stroke Disease of the Elderly}

A stroke is defined as a localized neurological deletion that is suddenly induced in poor blood flow. These strokes are called cerebrovascular stroke, and in terms of symptoms, the typical symptoms are classified as cerebral infarction and cerebral hemorrhage, caused by bleeding of the blood vessels [4-6]. These strokes are caused by rupture or blockage of the blood flow of the brain stem, resulting in sudden brain dysfunction, such as speech or movement disorders, sensory disorders, paralysis, or unconsciousness [24,25]. Cerebral infarction is classified into cerebral thrombosis, which is caused by blood clotting in cerebral blood vessels damaged by arteriosclerosis and blood clots; and cerebral embolism, which is caused by clotting of the blood vessels for large arteries, such as the heart and carotid arteries. Cerebral hemorrhage is represented by intracerebral hemorrhage and subarachnoid hemorrhage. Intracerebral hemorrhage is caused by spontaneous brain hemorrhage without external impact. Subarachnoid hemorrhage is a disease in which blood flows out into the subarachnoid space surrounding the brain, by the rupture of an aneurysm of the alveolar structure that grows in the blood vessel. Subarachnoid hemorrhage is reported to be a fatal disease, as more than $30 \%$ of the patients die before they arrive at the emergency medical center or hospital. In Korea, the health-screening information of the National Health Insurance Corporation (NHIS) [26], Lee et al. [27] developed a stroke prediction model after 10 years, using age, drinking, systolic and diastolic blood pressure, diabetes, LDL cholesterol, HDL cholesterol, total cholesterol, et al. However, since these studies are identical to the Framingham Heart's model and predict the stroke risk index in the next 10 years, they do not apply to the real world [16,27]. In addition, it does not take into account some of the major risk factors for stroke, and has a limitation, in that it does not consider the competition risk, such as the possibility of death other than by stroke. According to the recent research literature, the stroke should be accurately identified within $3 \mathrm{~h}$, including the type and location of the stroke, and how much damage has occurred. It is also important to send the patient to a hospital or emergency medical center, so that they can receive the appropriate treatment within $3 \mathrm{~h}$, known as the golden time.

\subsection{Research Subjects and Methods}

In this paper, the NIHSS to be used in the experiment were collected at the emergency medical center of Chungnam National University Hospital in Korea. Data collection for stroke patients was conducted within 3 days of the definite diagnosis date and data was collected from 287 patients from 2015 to 2017. Experimental data collected after 3 days of stroke definite diagnosis date that 16 patients (no stroke symptoms) with a value of 0 for NIHSS were included, indicating no specific stroke symptoms. We selected 227 NIHSS data from the finally selected clinical trial data, excluding 44 patients with outlier or missing values. Experimental patients were older than 65 years consisting of 117 men and 110 women. Table 1 shows the results of a standard deviation analysis for age. Table 2 below shows the distribution of variables for 287 subjects diagnosed with stroke.

The following Tables 3-5 shows the distribution of data measured in the emergency room for stroke patients. Table 3 shows the statistical distribution of normal and abnormal inclusion of blood pressure and blood test lists of stroke patients measured in the emergency room [28]. Tables 4 and 5 
below describes the statistical distribution of normal and abnormal conditions, such as emergency chemical, coagulation, and urinalysis test lists [29].

Table 1. Age-based standard deviation analysis.

\begin{tabular}{cccccc}
\hline Gender & Patients $(N)$ & Mean & Standard Deviation & Maximum & Minimum \\
\hline Male & 117 & 74.44 & 6.775 & 90 & 65 \\
Female & 110 & 77.82 & 6.661 & 99 & 65 \\
\hline
\end{tabular}

Table 2. Baseline characteristics for subjects $(N=287)$.

\begin{tabular}{|c|c|c|c|}
\hline Characteristics & No & Characteristics & No \\
\hline Gender & & Lesions & \\
\hline Male & 149 & Infarction & \\
\hline Female & 138 & Anterior Cerebral Artery & 7 \\
\hline Age & & Middle Cerebral Artery & 164 \\
\hline $65 \sim 69$ & 63 & Posterior Cerebral Artery & 25 \\
\hline $70 \sim 79$ & 120 & Basilar artery, Vertebral artery & 76 \\
\hline $80 \sim 89$ & 84 & Hemorrhage & \\
\hline$\geq 90$ & 8 & Cortex & 4 \\
\hline Causes & & Basal ganglion & 2 \\
\hline Infarction & 267 & Thalamus & 4 \\
\hline Hemorrhage & 16 & Brain stem & 2 \\
\hline Transient ischemic attacks & 4 & Cerebellum & 1 \\
\hline History & & Others & 3 \\
\hline Hypertension & 179 & NIHSS & \\
\hline Diabetes & 79 & 0 (No Stroke Symptoms) & 16 \\
\hline Previous stroke & 46 & 1 4 (Minor Stroke) & 149 \\
\hline Cardiovascular disease & 20 & 5 15 (Moderate Stroke) & 88 \\
\hline Nothing & 87 & 16 20 (Moderate to Severe Stroke) & 11 \\
\hline Symptom & & 21 42 (Severe Stroke) & 8 \\
\hline Weakness & 182 & ECG & \\
\hline Dysarthria & 113 & Normal ECG & 108 \\
\hline Aphasia & 26 & Abnormal ECG & 154 \\
\hline Decreased consciousness & 40 & Borderline ECG & 18 \\
\hline Facial palsy & 21 & & \\
\hline Headache & 12 & & \\
\hline Dizziness & 42 & & \\
\hline Paresthesia & 12 & & \\
\hline
\end{tabular}

Table 3. Characteristics of the emergency room for stroke patients.

\begin{tabular}{cccc}
\hline Vital Sign $(\mathrm{N}=\mathbf{2 8 6})$ & Mean $\pm \mathrm{SD}$ & Normal \\
Systolic Blood Pressure $(\mathrm{mmHg})$ & $154 \pm 17.5$ & $<120 \mathrm{mmHg}$ \\
Diastolic Blood Pressure $(\mathrm{mmHg})$ & $84 \pm 12.5$ & $<80 \mathrm{mmHg}$ \\
Pulse (beat $/ \mathrm{min})$ & $79 \pm 14.4$ & $80-100$ \\
Respiration Rate $(\# / \mathrm{min})$ & $20 \pm 2$ & \multicolumn{2}{c}{$36.1^{\circ} \mathrm{C}-37.2{ }^{\circ} \mathrm{C}$} \\
Body Temperature $\left({ }^{\circ} \mathrm{C}\right)$ & $37 \pm 0$ & Normal & More Than Normal \\
\hline Blood Pressure Test $(\mathrm{N}=\mathbf{2 8 6})$ & Less Than Normal & 87 & 199 \\
Systolic Blood Pressure $(\mathrm{mmHg})$ & 0 & 178 & 100 \\
Diastolic Blood Pressure $(\mathrm{mmHg})$ & 8 & Normal & More Than Normal \\
\hline Emergency Blood Test $(\mathrm{N}=\mathbf{2 8 4})$ & Less Than Normal & 233 & 48 \\
WBC $\left(10^{3} / \mathrm{uL}\right)$ & 3 & 158 & 1 \\
$\mathrm{RBC}\left(10^{6} / \mathrm{uL}\right)$ & 125 & 218 & 9 \\
$\mathrm{Hb}(\mathrm{g} / \mathrm{dL})$ & 57 & 182 & 36 \\
$\mathrm{Hct}(\%)$ & 66 & 249 & 4 \\
Platelet $\left(10^{3} / \mathrm{uL}\right)$ & 31 & &
\end{tabular}


Table 3. Cont.

\begin{tabular}{cccc}
\hline MCV (fL) & 5 & 264 & 15 \\
MCH (pg) & 3 & 281 & 0 \\
MCHC (g/dL) & 1 & 280 & 3 \\
MPV (fl) & 1 & 262 & 21 \\
Seg.Neutro (\%) & 1 & 211 & 72 \\
Lymphocyte (\%) & 80 & 200 & 4 \\
Monocyte (\%) & 19 & 246 & 19 \\
Eosinophil (\%) & 0 & 282 & 2 \\
Basophil (\%) & 0 & 284 & 0 \\
\hline
\end{tabular}

Table 4. Characteristics of the emergency chemical test.

\begin{tabular}{cccc}
\hline $\begin{array}{c}\text { Emergency Chemical } \\
\text { Test }(\boldsymbol{N}=\mathbf{2 6 1})\end{array}$ & Less Than Normal & Normal & More Than Normal \\
\hline TP(n) (g/dL) & 47 & 211 & 3 \\
Albumin $(\mathrm{g} / \mathrm{dL})$ & 197 & 64 & 0 \\
Glucose $(n)(\mathrm{mg} / \mathrm{dL})$ & 1 & 105 & 155 \\
$\mathrm{~TB}(n)(\mathrm{mg} / \mathrm{dL})$ & 12 & 235 & 14 \\
T.chol $(n)(\mathrm{mg} / \mathrm{dl})$ & 21 & 194 & 46 \\
$\mathrm{AST}(\mathrm{GOT})(\mathrm{U} / \mathrm{L})$ & 0 & 250 & 11 \\
$\mathrm{ALT}(\mathrm{GPT})(\mathrm{U} / \mathrm{L})$ & 0 & 252 & 9 \\
$\mathrm{ALP}(\mathrm{U} / \mathrm{L})$ & 6 & 251 & 4 \\
$\mathrm{CK}(\mathrm{CPK})(\mathrm{U} / \mathrm{L})$ & 55 & 196 & 10 \\
$\mathrm{UN}(\mathrm{U} / \mathrm{L})$ & 3 & 188 & 70 \\
$\mathrm{Cr}(\mathrm{mg} / \mathrm{dL})$ & 20 & 121 & 120 \\
Na $(\mathrm{mEq} / \mathrm{L})$ & 0 & 230 & 31 \\
$\mathrm{~K}(\mathrm{mEq} / \mathrm{L})$ & 13 & 247 & 1 \\
$\mathrm{CI}(\mathrm{mEq} / \mathrm{L})$ & 12 & 229 & 20 \\
$\mathrm{P}(\mathrm{mEq} / \mathrm{L})$ & 24 & 235 & 1 \\
Tca $(\mathrm{mg} / \mathrm{dL})$ & 68 & 192 & 50 \\
CRP $(\mathrm{mEq} / \mathrm{L})$ & 0 & 211 & 17 \\
CK-MB $(\mathrm{ng} / \mathrm{mL})$ & 7 & 237 & 28 \\
Troponin I (ng/mL) & 12 & 223 & \\
\hline
\end{tabular}

Table 5. Characteristics of the emergency coagulation and urinalysis test.

\begin{tabular}{cccc}
\hline $\begin{array}{c}\text { Emergency Coagulation } \\
\text { Test }(\boldsymbol{N}=\mathbf{2 8 1})\end{array}$ & Less Than Normal & Normal & More Than Normal \\
\hline aPTT (sec) & 13 & 264 & 4 \\
PT (sec) & 140 & 130 & 11 \\
PT (\%) & 7 & 274 & 0 \\
PT(INR) (ratio) & 0 & 280 & 1 \\
\hline Emergency Urinalysis & Less Than Normal & Normal & More Than Normal \\
Test ( $\boldsymbol{N}$ 161) & 1 & 148 & 12 \\
SG & 0 & 161 & 0 \\
pH & 0 & 125 & 36 \\
RBC (HPF) & 0 & 127 & 1 \\
WBC (HPF) & 0 & 160 & \\
Sq.epi.cell (HPF) & & & \\
\hline
\end{tabular}

The collected NIHSS data includes 17 features and age information in a medical examination, as shown in Table 5 below. All stroke patients were subjected to a pre-defined measurement scenario in a separate room in the emergency center, and NIHSS feature values were accurately assessed according to patient response. In this study, we scored the patients based on the NIHSS, and the scores for each item were analyzed using the Spearman correlation coefficient. In addition, the Spearman correlation coefficients $(r h o)$ for each NIHSS feature were statistically verified (see Table 6 below). 
Table 6. The Spearman correlation coefficients $(r h o)$ for each National Institutes of Health Stroke Scale (NIHSS) feature of the Korean elderly.

\begin{tabular}{|c|c|c|c|c|c|c|c|c|c|c|c|c|c|c|c|c|c|}
\hline Instructions & $1 a$ & $1 \mathrm{~b}$ & 1c & 2 & 3 & 4 & $5 a$ & $5 b$ & $6 a$ & $6 b$ & 7 & 8 & 9 & 10 & 11 & $12 a$ & $12 b$ \\
\hline $\begin{array}{c}\text { 1a. Level of } \\
\text { Consciousness }\end{array}$ & 1.00 & & & & & & & & & & & & & & & & \\
\hline $\begin{array}{l}\text { 1b. LOC } \\
\text { Questions }\end{array}$ & $0.420^{* *}$ & 1.00 & & & & & & & & & & & & & & & \\
\hline $\begin{array}{l}\text { 1c. LOC } \\
\text { Commands }\end{array}$ & $\underset{* *}{0.502}$ & $\underset{* *}{0.785}$ & 1.00 & & & & & & & & & & & & & & \\
\hline 2. Best Gaze & $\underset{* *}{0.400}$ & $\underset{* *}{0.209}$ & $309^{* *}$ & 1.00 & & & & & & & & & & & & & \\
\hline 3. Visual & -0.027 & 0.021 & 0.052 & 0.014 & 1.00 & & & & & & & & & & & & \\
\hline 4. Facial Palsy & 0.053 & 0.101 & 0.096 & $\underset{* *}{0.250}$ & $\underset{*}{-0.138}$ & 1.00 & & & & & & & & & & & \\
\hline $\begin{array}{l}\text { 5a. Motor Arm } \\
\text { (Left) }\end{array}$ & $\underset{* *}{0.236}$ & $\underset{* *}{0.389}$ & $\underset{* *}{0.456}$ & $\underset{* *}{0.174}$ & 0.033 & 0.102 & 1.00 & & & & & & & & & & \\
\hline $\begin{array}{l}\text { 5b. Motor Arm } \\
\text { (Right) }\end{array}$ & $\underset{* *}{0.337}$ & 0.068 & $0.143 *$ & $\underset{* *}{0.382}$ & -0.114 & $\underset{* *}{0.191}$ & $\underset{* *}{-0.21}$ & 1.00 & & & & & & & & & \\
\hline $\begin{array}{l}\text { 6a. Motor Leg } \\
\text { (Left) }\end{array}$ & $\underset{* *}{0.228}$ & $\underset{* *}{0.332}$ & $\underset{* *}{0.408}$ & $\underset{* *}{0.182}$ & 0.018 & 0.103 & $\underset{* *}{0.834}$ & $\underset{* *}{-0.22}$ & 1.00 & & & & & & & & \\
\hline $\begin{array}{l}\text { 6b. Motor Leg } \\
\text { (Right) }\end{array}$ & $\underset{* *}{0.342}$ & 0.053 & 0.125 & $\underset{* *}{0.375}$ & -0.040 & $\underset{* *}{0.176}$ & $\underset{* *}{-0.21}$ & $\begin{array}{c}0.847 \\
* *\end{array}$ & $\underset{* *}{-0.21}$ & 1.00 & & & & & & & \\
\hline 7. Limb Ataxia & -0.039 & -0.063 & -0.114 & -0.053 & 0.045 & -0.071 & $\underset{*}{-0.158}$ & -0.117 & $\underset{*}{-0.167}$ & $\underset{*}{-0.132}$ & 1.00 & & & & & & \\
\hline 8. Sensory & -0.038 & -0.22 & $\underset{*}{-0.139}$ & 0.010 & -0.112 & 0.023 & $\underset{*}{-0.138}$ & 0.083 & -0.101 & 0.013 & -0.067 & 1.00 & & & & & \\
\hline 9. Best Language & $\begin{array}{c}0.349 \\
* *\end{array}$ & $\underset{* *}{0.770}$ & $\underset{* *}{0.757}$ & $\underset{* *}{0.206}$ & 0.072 & 0.107 & $\begin{array}{c}0.480 \\
* *\end{array}$ & -0.034 & $\underset{* *}{0.403}$ & -0.052 & -0.080 & $-0.162^{*}$ & 1.00 & & & & \\
\hline 10. Dysarthria & $\underset{* *}{0.233}$ & $\underset{* *}{0.200}$ & $\underset{* *}{0.313}$ & $\underset{* *}{0.285}$ & 0.010 & $\underset{* *}{0.291}$ & $\underset{* *}{0.291}$ & 0.095 & $\underset{* *}{0.263}$ & $0.140 *$ & -0.090 & -0.014 & $\underset{* *}{0.292}$ & 1.00 & & & \\
\hline $\begin{array}{l}\text { 11. Extinction } \\
\text { and Inattention }\end{array}$ & $0.148^{*}$ & $\underset{* *}{0.183}$ & $\underset{* *}{0.184}$ & $\underset{* *}{0.534}$ & -0.033 & $\underset{* *}{0.336}$ & 0.056 & $\underset{* *}{0.396}$ & 0.056 & $\underset{* *}{0.379}$ & -0.094 & 0.028 & $0.138^{*}$ & $\underset{* *}{0.251}$ & 1.00 & & \\
\hline $\begin{array}{l}\text { 12. Distal Motor } \\
\text { Function (Left) }\end{array}$ & -0.033 & 0.029 & -0.127 & 0.017 & 0.079 & -0.098 & -0.197 & -0.219 & -0.27 & -0.139 & -0.059 & -0.119 & -0.051 & 0.49 & -0.069 & 1.00 & \\
\hline $\begin{array}{l}\text { 12. Distal Motor } \\
\text { Function (Right) }\end{array}$ & -0.026 & -0.103 & 0.113 & 0.014 & 0.059 & -0.058 & -0.133 & 0.094 & -0.127 & -0.069 & -0.069 & -0.21 & 0.038 & 0.86 & -0.033 & 0.071 & 1.00 \\
\hline
\end{tabular}




\subsection{Machine Learning in Stroke Analysis and Disease Prediction}

According to a recent research, data mining and machine learning methodologies have an important role in the diagnosis and prediction of diseases in the healthcare application [30-35]. Recently, various studies have been published on the prediction and analysis of brain diseases, using data mining and machine learning methodology [30-34]. A decision tree is a method of decision support tool that shows the various rules and their results in a tree structure. A decision tree is a tree-like structure, where each internal node denotes a test on a feature, each current node describes a decision result, and leaf node or each terminal node holds a class label. Typically, the topmost node in the tree is the root node. These decision tree methods are intuitive in predicting results, and generate rules logically and conveniently [35-38]. ID3 is a representative algorithm of decision tree, and the high-rank node has a disadvantage in selecting a feature having a wide range of values [37]. Therefore, in this paper, we introduced the C4.5 decision tree algorithm that alleviates these disadvantages [38]. C4.5 is the most advanced decision tree algorithm, and has proved that classification and prediction performance is advanced in many existing researches [37-39]. In C4.5 decision tree, each node in the tree has an association between cases, and this case, are assigned weights, considering the unknown feature values.

When there is an arbitrary set $Y$, the information gain value of attribute a is calculated as follows. Now, consider a similar measurement after $Y$ has been partitioned in accordance with the $n$ outcomes. The expected information requirement can be found as the weighted sum over subsets, as gain or $\operatorname{gain}(Y)$. Here, $Y i$ means the $i$-th set of arbitrary set $Y$. The expected information needed to classify a tuple in $Y$ is given by

$$
\operatorname{gain}=\operatorname{info}(Y)-\sum_{i=1}^{n} \frac{\left|Y_{i}\right|}{|Y|} \times \operatorname{info}\left(Y_{i}\right)
$$

The quantity Equation (1) measures is the information that is gained by partitioning $Y$ in accordance with the test subsets. The gain criterion, selects a test subset to maximize this information gain.

$$
\operatorname{info}(Y)=-\sum_{j=1}^{\text {NClass }} \frac{\text { freq }\left(C_{j}, Y\right)}{|Y|} \times \log _{2}\left(\frac{\text { freq }\left(C_{j}, Y\right)}{|Y|}\right)
$$

where is the entropy function. Here, freq $(C j, Y)$ means the frequency of appearance of the $j$-th class in arbitrary set $Y$. The information gain measure is biased toward tests with many outcomes. Apply a kind of normalization to the information gain using the 'split information' value defined analogously with info $(Y)$ calculated in Equation (2) above. By analogy with the definition of info( $Y$ ), we have

$$
\text { Split info }(Y)=-\sum_{i=1}^{n} \frac{\left|Y_{i}\right|}{|Y|} \times \log _{2}\left(\frac{\left|Y_{i}\right|}{|Y|}\right)
$$

This represents the potential information generated by dividing $Y$ into $n$ subsets, whereas the information gain measures the information relevant to classification that arises from the same division. It is easy to see that the gain and gain ratios are zero if we select the discrete attribute or feature at the parent ancestor node. Therefore, the C 4.5 decision tree does not calculate the value of the information gain for this attribute or feature. In Equation (3) above, $Y$ is divided into subset of $n$, to generate a potentially inherent information value, but the information gain value calculates information related to classification and prediction occurs on the same node. The gain ratio is defined as

$$
\operatorname{Gain} \operatorname{ratio}(Y)=\frac{\operatorname{gain}(Y)}{\operatorname{Split} \operatorname{info}(Y)}
$$


The gain ratio $(Y)$ obtained from the above Equation (4) represents the ratio value of information generated by partitioning [38]. The feature with the highest gain ratio $(Y)$ value at that node is the partitioned value, i.e., that appears helpful for classification and prediction.

\section{A Stroke Severity Prediction and In-Depth Analysis System Using NIHSS}

In this section, we propose a new system for real-time predicting and in-depth analysis the severity of strokes based on NIHSS features. Figure 1, below, illustrates the overall architecture of our proposed system. The system consists of NIHSS features collection module, medical data server, real-time stroke severity prediction and analysis, cloud-based stroke monitoring and emergency alarm modules. Our medical data center has real-time patient NIHSS data and a health-screening information update module and the training and prediction server module for real-time stroke severity, while each patient has a smart device in a server and various healthcare sensors (smart phone, healthcare device, etc.) to collect the NIHSS 17 features and age. The NIHSS-based stroke severity prediction and in-depth analysis system proposed in this paper is used for execution and service according to the following procedure.

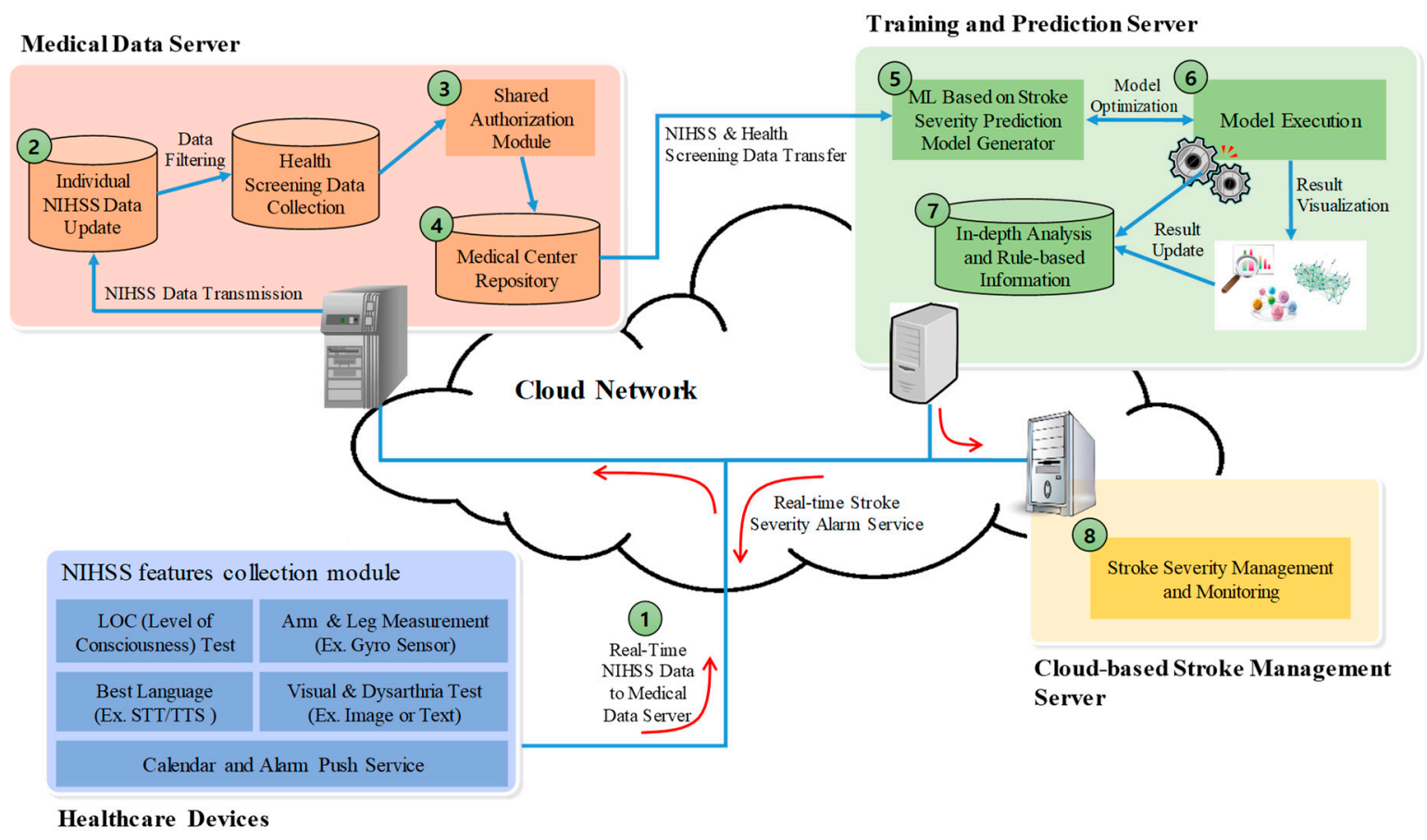

Figure 1. The proposed system architecture for stroke severity prediction and analysis of the elderly.

1. Elderly users of stroke severity prediction and in-depth analysis applications collect real-time NIHSS data using various healthcare devices. The collected NIHSS data is transmitted to the medical network server through a wired or wireless communication network.

2. The medical data server updates in real-time the NIHSS data collected by individual patients. In addition, the individual NIHSS data and health medical examination information are saved and transmitted to the health-screening information collection in the repository.

3. Individual health screening and NIHSS data stored in the database of health screening data collection should be updated after filtering outlier or missing values. The data collected and stored in the database of health screening data collection is transmitted, in real-time, to the individual patient shared authentication module.

4. The patient data and information collected at the medical center repository are forwarded to a module that generates a stroke severity learning model. Stroke severity learning model using NIHSS data analyzes patient-specific NIHSS data collected in real-time to determine the severity 
of stroke risk. In addition, to predict and analyze more accurate and faster stroke severity, important features in the medical center repository are selected or reduced, to ensure optimal prediction accuracy.

5. The stroke severity learning and prediction model of the present system can select various machine learning algorithms and perform learning repeatedly. We also provide models that provide optimal prediction accuracy and analysis information through repetitive learning and performance verification.

6. When NIHSS data collected in real-time is executed in the predictive model, it is possible to determine the severity of stroke and in-depth analysis information. In addition, the stroke severity prediction value and analysis information is provided to the system administrator. System administrators can provide alarms for stroke risk to patients and their families.

7. In general, machine learning is a mechanism that generates a prediction model through random learning, and classifies and predicts real-time stroke severity by class using actual data. In this system, semantic analysis and in-depth analysis algorithms, such as the C4.5 decision tree, Bayesian, logistic regression, and random forest, which are represented by an analytical model rather than a black box of machine learning, can be utilized.

8. Stroke severity management and monitoring server in the cloud-based environment receives the prediction and in-depth analysis of the severity for each patient. In addition, the stroke severity value and the analysis information are transmitted to the patient and the medical doctor, to execute an alarm application for emergency situations.

\section{Experiment and Analysis}

\subsection{Experimental Environment and Considerations}

In this study, we verified the performance using Hall et al. [40] in a Java-based data mining package and the Shi et al. [41] for extreme gradient boosting. Our experimental data used in this research were collected at the emergency medical center of Chungnam National University Hospital in Korea. Clinical data collection for stroke patients was conducted within 3 days of the definite diagnosis date, and data was collected from 287 patients from 2015 to 2017. We selected 227 HINSS data from the finally selected clinical trial data, excluding 44 patients with outlier or missing values. Experimental patients were older than 65 years consisting of 117 men and 110 women. We divided the research into 17 features, such as the Level of Consciousness (LOC), LOC questions, LOC commands, best gaze, visual, facial palsy, motor leg (left), motor leg (right), motor arm (left), motor arm (right), sensory, dysarthria, best language, limb ataxia, extinction and inattention (formerly neglect), distal motor function (left), and distal motor function (right) of the NIHSS features. Additionally, by including the age in the experimental data, the severity of a stroke patient was measured using a total of 18 features. All stroke patients were subjected to a pre-defined measurement scenario in a separate room in the emergency center, and NIHSS feature values were evaluated according to patient response. Each patient had a normal NIHSS score close to zero, but an elderly patient with a higher score means severe stroke severity. The individual scores from each item are summed to calculate a patient's total NIHSS score. The maximum possible score is 42 , and the minimum score is 0 . The severity index of the stroke is classified into five classes according to the NIHSS score, as shown in Table 7 below. In a total of 227 stroke patient class distribution, 127 patients with 'minor stroke', 84 patients with 'moderate stroke', 10 patients with 'moderate to severe stroke', and six patients with 'severe stroke'. 
Table 7. Stroke severity class of NIHSS.

\begin{tabular}{cc}
\hline NIHSS Score & Stroke Severity \\
\hline 0 & No Stroke Symptoms \\
$1 \sim 4$ & Minor Stroke \\
$5 \sim 15$ & Moderate Stroke \\
$16 \sim 20$ & Moderate to Severe Stroke \\
$21 \sim 42$ & Severe Stroke \\
\hline
\end{tabular}

Table 8 below shows the actual values for age and NIHSS 17 features for each patient at Chungnam National University Hospital.

Table 8. An example of the NIHSS score.

\begin{tabular}{cccccccccccccccccc}
\hline Age & $\mathbf{1 a}$ & $\mathbf{1 b}$ & $\mathbf{1 c}$ & $\mathbf{2}$ & $\mathbf{3}$ & $\mathbf{4}$ & $\mathbf{5 a}$ & $\mathbf{5 b}$ & $\mathbf{6 a}$ & $\mathbf{6 b}$ & $\mathbf{7}$ & $\mathbf{8}$ & $\mathbf{9}$ & $\mathbf{1 0}$ & $\mathbf{1 1}$ & $\mathbf{1 2 a}$ & $\mathbf{1 2 b}$ \\
\hline 68 & 0 & 0 & 0 & 0 & 0 & 1 & 0 & 4 & 0 & 4 & 0 & 0 & 0 & 1 & 0 & 1 & 0 \\
86 & 2 & 1 & 1 & 1 & 0 & 1 & 1 & 2 & 0 & 1 & 1 & 0 & 2 & 0 & 1 & 0 & 1 \\
75 & 0 & 0 & 0 & 0 & 0 & 2 & 1 & 0 & 0 & 1 & 0 & 0 & 0 & 0 & 1 & 0 & 0 \\
92 & 2 & 2 & 2 & 0 & 0 & 0 & 3 & 1 & 0 & 3 & 2 & 0 & 0 & 2 & 2 & 1 & 0 \\
65 & 0 & 1 & 0 & 1 & 1 & 0 & 2 & 1 & 0 & 1 & 2 & 0 & 0 & 1 & 1 & 0 & 0 \\
& & & & & & & & & & & & & & & & & \\
$\cdots$ & $\cdots$ & $\cdots$ & $\ldots$ & $\ldots$ & $\ldots$ & $\ldots$ & $\cdots$ & $\ldots$ & $\ldots$ & $\cdots$ & $\cdots$ & $\cdots$ & $\cdots$ & $\cdots$ & $\cdots$ & $\cdots$ & $\cdots$ \\
\hline
\end{tabular}

\subsection{An In-Depth Analysis on the Stroke Severity Using Machine Learning}

This experiment classifies and predicts the stroke severity using C4.5 decision tree algorithm, which is representative of machine learning methodology. In this experiment, four classes of stroke severity were classified, and their performance was verified. In this study, a total of 227 clinical NIHSS data were used, and a machine learning model was generated by randomly extracting data from 182 patients. We performed the tests with NIHSS data from 45 patients-the remaining $20 \%$ who did not participate in the learning model generation. In order to measure the classification and prediction accuracy of the proposed system, we used recall and precision as performance evaluation [42-44]. For each patient's NIHSS feature, the data correctly classified into that class is shown as a true positive (TP). Next, the data of misclassification patient are expressed as false positives (FP). The patient's NIHSS data from a class that are falsely labeled as belonging to another class are denoted as false negatives (FN). Recall represents the ratio of TP to TP + FN. Recall means the unit of data that is misclassified by each class. Precision represents the ratio of TP to TP + FP. Precision means the number of correctly identified data for each class. Overall accuracy is the percentage of correctly identified data for each class divided by data elements of all classes.

$$
\begin{gathered}
\text { Recall }=\frac{T P}{T P+F N} \\
\text { Precision }=\frac{T P}{T P+F P} \\
\text { Overall accuracy }=\frac{\text { Sum of the element of TP in each class }}{\text { Total element }}
\end{gathered}
$$

The accuracy of the stroke severity classification and prediction was measured by experimental comparison with C4.5 decision tree and random forest, logistic regression, classification and regression tree (CART), extreme gradient boosting (XgBoost), naïve Bayes, artificial neural network (ANN), multi-class support vector machine (SVM), and one-class support vector data description (SVDD), a delegate advanced research methodology. We used the correlation feature selection (CFS) described to select the optimal feature subset for an accurate classification and prediction of each class representing stroke severity [42-45]. The feature subset selected by the CFS is as follows: level of consciousness, 
LOC questions, LOC commands, facial palsy, motor arm (right), motor leg (left), motor leg (right), best language, dysarthria, extinction and inattention (formerly neglect). Table 9 compares the results of using only 11 features selected by the CFS and the classification and prediction results using all 18 features.

Table 9. The performance measurement for stroke severity prediction accuracy (\%).

\begin{tabular}{ccccc}
\hline \multirow{2}{*}{ Performance Measure } & \multicolumn{2}{c}{ All Features Used } & \multicolumn{2}{c}{$\begin{array}{c}\text { Correlation Feature Selection } \\
\text { (CFS) (11 Features Used) }\end{array}$} \\
\cline { 2 - 5 } Methods & Recall & Precision & Recall & Precision \\
\hline C4.5 decision tree & 91.1 & 93.3 & 88.9 & 87.5 \\
Random forest & 88.9 & 90.7 & 89.4 & 90.3 \\
Logistic regression & 88.9 & 90.9 & 86.7 & 86.6 \\
CART & 89.8 & 92.1 & 88.4 & 89.8 \\
XgBoost & 88.6 & 89.6 & 88.9 & 91.2 \\
Naive Bayes & 84.4 & 88.1 & 82.2 & 86.7 \\
ANN(MLP) & 86.6 & 85.7 & 91.1 & 92.3 \\
Multi-class SVM & 82.2 & 84.5 & 80.0 & 82.1 \\
One-class SVM & 80.0 & 85.4 & 84.4 & 87.8 \\
\hline
\end{tabular}

The results of the experiment show that the classification and prediction accuracy was higher in the case of using all the features in the initial stroke severity evaluation than in the case of using the optimal 11 feature subset [45-47]. For this reason, rather than removing the NIHSS features that are rarely used or duplicated by the CFS [43,46,47], important feature selection was made using the information gain or gain ratio of the decision tree model, such as $\mathrm{C} 4.5$ or random forest algorithms. In the C4.5 decision tree algorithm, the parameters are set and tested with the confidence threshold for pruning at 0.5 , the seed value is 1 , and the minimum number of leaf nodes is 1 . In the random forest algorithm, the number of trees was set to 100, the iteration was 200 times, the seed value was set to 1 , the tree showed stable performance at least 100, and the iteration was 150 times or more. For logistic regression, the batch size was set to 100 and the ridge parameter value was set to 1.0E-8. In CART, the impurity was set as the splitting rule that best distinguishes the distribution of target variables. In addition, the impurity was set to the gini, the confidence threshold for pruning was 0.5 , and the stopping rule was set to end when impurity no longer dropped. In XgBoost, we experimented by setting the learning rate value to 0.25 , the learning rate value is set to 0.01 , the gamma value to 0 , the tree depth to 12 , and the subsampling value to 1 . The learning rate value showed relatively stable accuracy from 0.01 to 0.001 , and, in this paper, the optimal stroke severity prediction accuracy when set to 0.01 . Naïve Bayes showed the best performance when the batch size was set to 100 and the number of decimal places was set to 2 . ANN was tested by setting the learning rate to 0.03 , the momentum to 0.2 , the number of hidden layers to 2 , and the number of nodes to the hidden layer to 24 . In the two SVM algorithms, the trade-off constant $C$ in the Gaussian Kernel function is set to $C=0.1$ in both phases. The parameter $\sigma$ in the Gaussian Kernel function are chosen as 0.02 and 0.03 in the multi-class SVM and one-class SVM of our experiment, respectively. Furthermore, the C4.5 decision tree and random forest algorithms have the advantage of being able to perform an interpretative analysis of stroke severity and rule-based analysis, unlike previous studies. In addition, it has the advantage of predicting stroke severity more quickly and accurately, and executing system operations efficiently.

Figure 2 shows our performance evaluation of the overall accuracy. In our experiment, the health stroke scale classification and prediction for elderly were used with different datasets. Figure 2 illustrates the accuracy of stroke severity prediction with all features and only 11 attributes based on CFS. In particular, when generating a predictive model by fixing a training set and a test set, an overfitting problem that works well only in a test set may occur. Therefore, in this experiment, the performance is verified by constructing a training set and a test set with 10-fold and 20-fold cross validation to minimize overfitting, while repeatedly tuning the stroke severity predictive model. As a 
result, when using all NIHSS features, the C4.5 decision tree algorithm showed the highest classification and prediction accuracy in recall and precision.

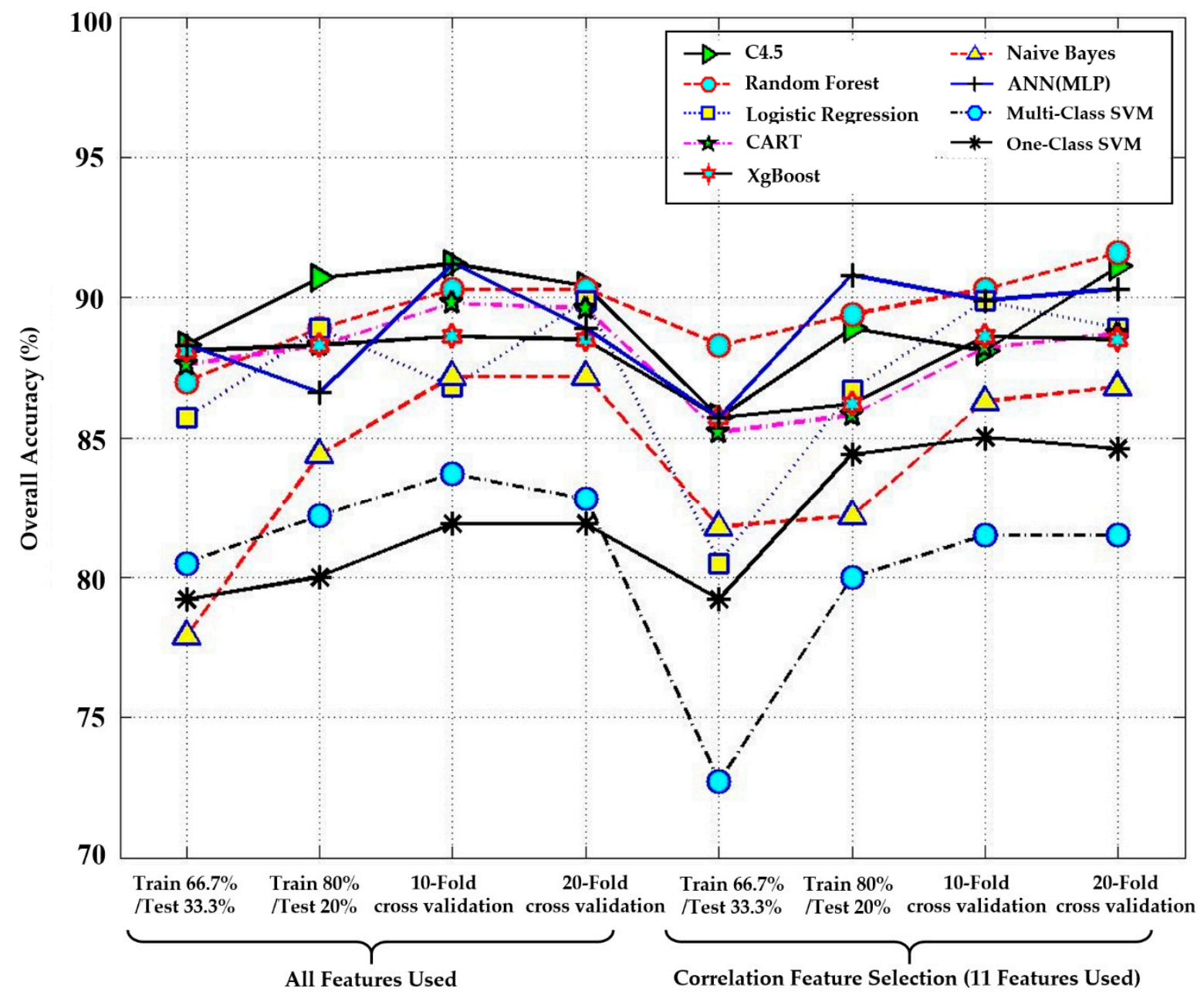

Figure 2. The Performance evaluation of the overall accuracy with various datasets.

Table 10 below shows the C4.5-based classification and overall accuracy of class of stroke severity. In this experiment, a total of 227 clinical NIHSS data were used, and a machine learning model was generated by randomly extracting data from 182 patients. We performed the tests with NIHSS data from 45 patients, the remaining $20 \%$ who did not participate in the learning model generation. Each class consisted of 24 minor stroke severity patients, 19 moderate stroke severity patients, one moderate-to-severe stroke patient and one severe stroke patient. The results of the test correctly found 22 out of 24 stroke severity patients in the 'minor stroke' class, 16 out of 19 stroke severity patients in the 'moderate stroke' class, and classified and predicted the 'moderate-to-severe stroke' and 'severe stroke' class for either patient (refer to Table 9). Experimental results show that the system enables this by reducing the patient NIH stroke scale measurement time and making the operation more efficient, with overall accuracy using the C4.5 algorithm of $91.11 \%$.

Table 10. The performance evaluation in stroke severity class classification and prediction.

\begin{tabular}{ccccc}
\hline Minor Stroke & Moderate Stroke & $\begin{array}{c}\text { Moderate to } \\
\text { Severe Stroke }\end{array}$ & Severe Stroke & Overall Accuracy \\
\hline $91.67 \%$ & $84.21 \%$ & $100 \%$ & $100 \%$ & $91.11 \%$ \\
\hline
\end{tabular}


Figure 3 shows a C4.5-based tree structure for the classification and prediction by stroke severity types of the Korean elderly. The figure shows than stroke severity types can be precisely classified with thirteen features. The twenty rules obtained from this experiment are shown in Figure 3 below (refer to Table 11).

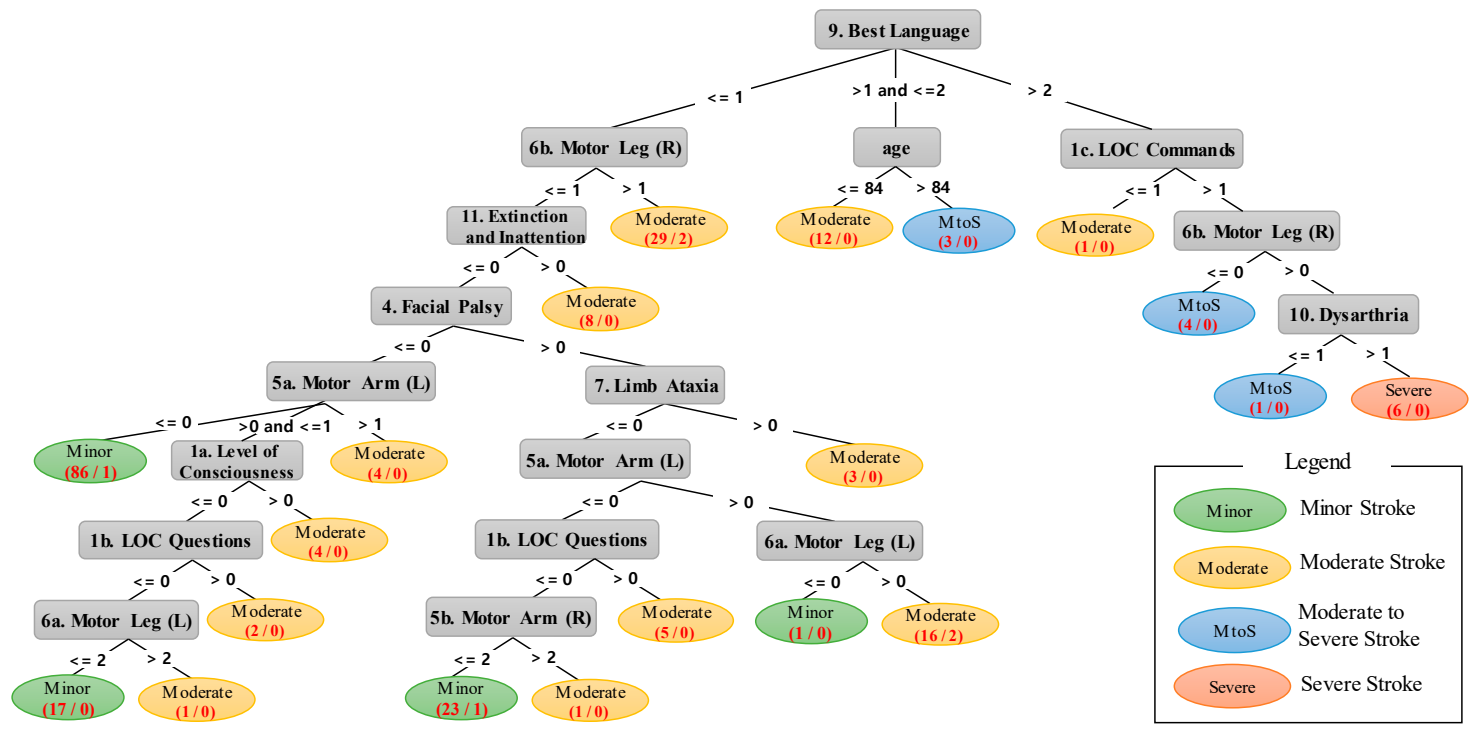

Figure 3. The C4.5-based tree structure for stroke severity types classification and prediction.

Table 11. The rules for stroke severity classification and in-depth analysis.

\begin{tabular}{|c|c|}
\hline Rule \# & A Rules and Analysis Results Found in Figure 3. \\
\hline 1 & IF $1<$ Best Language $\leq 2$ AND age $\leq 84$ THEN Moderate stroke. \\
\hline 2 & IF $1<$ Best Language $\leq 2$ AND age $>84$ THEN Moderate-to-severe stroke. \\
\hline 3 & IF Best Language $>2$ AND LOC Commands $\leq 1$ THEN Moderate stroke. \\
\hline 4 & $\begin{array}{c}\text { IF Best Language }>2 \text { AND LOC Commands }>1 \text { AND Motor Leg (Right) } \leq 0 \text { Moderate-to-severe } \\
\text { stroke. }\end{array}$ \\
\hline 5 & $\begin{array}{c}\text { IF Best Language }>2 \text { AND LOC Commands }>1 \text { AND Motor Leg (Right) }>0 \text { AND Dysarthria } \leq \\
1 \text { THEN Moderate-to-severe stroke. }\end{array}$ \\
\hline 6 & $\begin{array}{l}\text { IF Best Language }>2 \text { AND LOC Commands }>1 \text { AND Motor Leg (Right) }>0 \text { AND Dysarthria }> \\
1 \text { THEN Severe stroke. }\end{array}$ \\
\hline 7 & IF Best Language $\leq 1$ AND Motor Leg (Right) $>1$ THEN Moderate stroke. \\
\hline 8 & $\begin{array}{c}\text { IF Best Language } \leq 1 \text { AND Motor Leg (Right) } \leq 1 \text { AND Extinction and Inattention }>0 \text { THEN } \\
\text { Moderate stroke. }\end{array}$ \\
\hline 9 & $\begin{array}{l}\text { IF Best Language } \leq 1 \text { AND Motor Leg }(\text { Right }) \leq 1 \text { AND Extinction and Inattention } \leq 0 \text { AND } \\
\text { Facial Palsy } \leq 0 \text { AND Motor Arm (Left) } \leq 0 \text { THEN Minor stroke. }\end{array}$ \\
\hline 10 & $\begin{array}{c}\text { IF Best Language } \leq 1 \text { AND Motor Leg (Right) } \leq 1 \text { AND Extinction and Inattention } \leq 0 \text { AND } \\
\text { Facial Palsy } \leq 0 \text { AND Motor Arm (Left) }>1 \text { THEN Moderate stroke. }\end{array}$ \\
\hline 11 & $\begin{array}{c}\text { IF Best Language } \leq 1 \text { AND Motor Leg (Right) } \leq 1 \text { AND Extinction and Inattention } \leq 0 \text { AND } \\
\text { Facial Palsy } \leq 0 \text { AND }>0 \text { Motor Arm (Left) } \leq 1 \text { AND Level of Consciousness }>0 \text { THEN } \\
\text { Moderate stroke. }\end{array}$ \\
\hline 12 & $\begin{array}{c}\text { IF Best Language } \leq 1 \text { AND Motor Leg }(\text { Right }) \leq 1 \text { AND Extinction and Inattention } \leq 0 \text { AND } \\
\text { Facial Palsy } \leq 0 \text { AND }>0 \text { Motor Arm (Left) } \leq 1 \text { AND Level of Consciousness }>0 \text { AND LOC } \\
\text { Questions }>0 \text { THEN Moderate stroke. }\end{array}$ \\
\hline
\end{tabular}


Table 11. Cont.

\begin{tabular}{cc}
\hline Rule \# & A Rules and Analysis Results Found in Figure 3. \\
\hline \multirow{2}{*}{13} & $\begin{array}{c}\text { IF Best Language } \leq 1 \text { AND Motor Leg (Right) } \leq 1 \text { AND Extinction and Inattention } \leq 0 \text { AND } \\
\text { Facial Palsy } \leq 0 \text { AND }>0 \text { Motor Arm (Left) } \leq 1 \text { AND Level of Consciousness }>0 \text { AND LOC } \\
\text { Questions } \leq 0 \text { Motor Leg (Left) } \leq 2 \text { THEN Minor stroke. }\end{array}$ \\
\hline \multirow{3}{*}{ IF Best Language $\leq 1$ AND Motor Leg (Right) $\leq 1$ AND Extinction and Inattention $\leq 0$ AND } \\
Facial Palsy $\leq 0$ AND $>0$ Motor Arm (Left) $\leq 1$ AND Level of Consciousness $>0$ AND LOC \\
Questions $\leq$ 0 Motor Leg (Left) $>2$ THEN Moderate stroke.
\end{tabular}

Our analysis of the rules in Table 11 of the C4.5 decision tree reveals that the stroke severity could be precisely classified and predicted with only 13 out of the 18 features that were defined in this system. Therefore, reducing the features used in the classification and prediction can not only improve the classification and prediction speed, but can also can improve the precision of the classification and prediction system.

\section{Conclusions}

In this paper, we propose a system to detect and in-depth analysis stroke severity of elderly aged over 65 years based on the NIHSS features, based on the C4.5 decision tree algorithm. The proposed stroke severity prediction and in-depth analysis system has the following advantages. First, our system that automatically classifies and analysis stroke severity into four classes using NIHSS features collected in real-time. Second, the system provides patients and their families with alarm information of stroke severity in real-time, so patients can receive medical center visits and emergency care. Third, the additional in-depth rules provided in C4.5 decision tree were analyzed, and semantic analysis was performed. Lastly, during the operation of an actual system, the proposed model uses only 13 features out of the 18 NIHSS features, including age, so that it can provide faster and more accurate service support. To summarize the advantages of our system, the NIHSS measurement time of the patient can be scientifically reduced. In addition, it can contribute to securing the golden time for emergency care of a patient and providing highly reliable services.

The in-depth analysis method applied in this paper to stroke severity and stroke disease prediction based on the C4.5 decision tree is innovative research, which has not previously been attempted, and offers considerable potential for future applications. However, decision tree algorithms, due to their nature, do not provide as complete a semantic analysis as the predictive model algorithm. Therefore, our future work will use association rule mining as a research tool for useful knowledge discovery 
and analysis, which is inherent in the mechanism for initial stroke assessment of disability and the recurrence prediction.

Author Contributions: Formal analysis, S.P. and H.L.; Funding acquisition, C.-S.P.; Methodology, S.P. and C.-S.P.; Software, J.Y.; Supervision, Y.S.L.; Validation, H.L.; Writing—original draft, J.Y.; Writing—review \& editing, Y.S.L. All authors have read and agreed to the published version of the manuscript.

Funding: This work was supported by the National Research Council of Science \& Technology (NST) grant by the Korea government (MSIP) (No. CRC-15-05-ETRI).

Conflicts of Interest: The authors declare no conflict of interest.

\section{References}

1. Statistics Korea. The Cause of Death Statistics in Koreans. Available online: Kosis.kr/eng/statisticsList/ statisticsListIndex.do?menuId=M_01_01\&vwcd=MT_ETITLE\&parmTabId=M_01_01\#SelectStatsBoxDiv (accessed on 25 June 2020).

2. Racosta, J.M.; Guglielmo, D.; Klein, F.R.; Riccio, P.M.; Giacomelli, F.M.; Toledo, M.E.G.; Cassará, F.P.; Tamargo, A.; Delfitto, M.; Sposato, L.A. Stroke severity score based on six signs and symptoms the 6 score-A simple tool for assessing stroke severity and in-hospital mortality. J. Stroke 2014, 16, 178-183. [CrossRef]

3. Lee, H.J.; Lee, J.S.; Choi, J.C.; Cho, Y.; Kim, B.J.; Bae, H.; Kim, D.; Ryu, W.; Cha, J.; Kim, D.H.; et al. Simple estimates of symptomatic intracranial hemorrhage risk and outcome after intravenous thrombolysis using age and stroke severity. J. Stroke 2017, 19, 229-231. [CrossRef] [PubMed]

4. Sengupta, A.; Rajan, V.; Bhattacharya, S. A statistical model for stroke outcome prediction and treatment planning. In Proceedings of the IEEE 38th Annual International Conference of the Engineering in Medicine and Biology Society (EMBC), Orlando, FL, USA, 16-20 August 2016; pp. 2516-2519.

5. Zhang, Q.; Xie, Y.; Ye, P.; Pang, G. Acute ischaemic stroke prediction from physiological time series patterns. J. Australas. Med. 2013, 6, 280-286. [CrossRef] [PubMed]

6. Chien, K.L.; Su, T.C.; Hsu, H.C.; Chang, W.T.; Chen, P.C.; Sung, F.C.; Chen, M.F.; Lee, Y.T. Constructing the prediction model for the risk of stroke in a Chinese population. Am. Heart Assoc. 2010, 41, 1858-1864. [CrossRef]

7. Brott, T.; Adams, H.P.; Olinger, C.P.; Marler, J.R.; Brasan, W.G.; Biller, J.; Spilker, J.; Holleran, R.; Eberle, R.; Hertzberg, V. Measurements of acute cerebral infarction: A clinical examination scale. Am. Heart Assoc. 1989, 20, 864-870. [CrossRef] [PubMed]

8. $\quad$ Lyden, P.; Brott, T.; Tilley, B.; Welch, K.M.; Mascha, E.J.; Levine, S.; Haley, E.C.; Grotta, J.; Marler, J. Improved reliability of the NIH Stroke Scale using video training: NINDS TPA stroke study group. Am. Heart Assoc. 1994, 25, 2220-2226. [CrossRef] [PubMed]

9. Meyer, B.C.; Lyden, P.D. The modified National Institutes of health stroke scale: Its time has come. J. Stroke 2009, 4, 267-273. [CrossRef]

10. Schlegel, D.; Kolb, S.J.; Luciano, J.M.; Tovar, J.M.; Cucchiara, B.L.; Liebeskind, D.S.; Kasner, S.E. Utility of the $\mathrm{NIH}$ stroke scale as a predictor of hospital disposition. Am. Heart Assoc. 2003, 34, 134-137. [CrossRef]

11. Yu, J.; Kim, D.; Park, H.; Chon, S.; Cho, K.H.; Kim, S.; Yu, S.; Park, S.; Hong, S. Semantic analysis of NIH stroke scale using machine learning techniques. In Proceedings of the 2019 International Conference on Platform Technology and Service (PlatCon), Jeju, Korea, 28-30 January 2019; pp. 82-86.

12. Lai, S.M.; Duncan, P.W.; Keighley, J. Prediction of functional outcome after stroke: Comparison of the orpington prognostic scale and the NIH stroke scale. Am. Heart Assoc. 1998, 29, 1838-1842. [CrossRef]

13. Powers, D.W. Assessment of the stroke patient using the NIH stroke scale. Emerg. Med. Serv. 2001, 30, 52-56.

14. Shrestha, S.; Poudel, R.S.; Khatiwada, D.; Thapa, L. Stroke subtype, age, and baseline NIHSS score predict ischemic stroke outcomes at 3 months a preliminary study from Central Nepal. J. Multidiscip. Healthc. 2015, 8, 443-448. [CrossRef] [PubMed]

15. Sartor, E.A.; Albright, K.; Boehme, A.K.; Morales, M.M.; Shaban, A.; Grotta, J.C.; Savitz, S.I.; Martin, S. The NIHSS score and its components can predict cortical stroke. J. Neurol. Disord. Stroke 2013, 2, 73-78.

16. Wolf, P.A.; D'Agostino, R.B.; Belanger, A.J.; Kannel, W.B. Probability of stroke: A risk profile from the Framingham study. Am. Heart Assoc. 1991, 22, 312-318. [CrossRef] [PubMed] 
17. D'Agostino, R.B.; Vasan, R.S.; Pencina, M.J.; Wolf, P.A.; Cobain, M.; Massaro, J.M.; Kannel, W.B. General cardiovascular risk profile for use in primary care. Am. Heart Assoc. 2008, 6, 743-753. [CrossRef]

18. Jee, S.H.; Park, J.W.; Lee, S.Y.; Nam, B.H.; Ryu, H.G.; Kim, S.Y.; Kim, Y.N.; Lee, Y.; Choi, S.M.; Yun, J.E. Stroke risk prediction model: A risk profile from the Korean study. J. Atheroscler. 2008, 197, 318-325. [CrossRef] [PubMed]

19. Wang, T.J.; Massaro, J.M.; Levy, D.; Vasan, R.S.; Wolf, P.A.; D'Agostino, R.B.; Larson, M.G.; Kannel, W.B.; Benjamin, E.J. A risk score for predicting stroke or death in individuals with new-onset atrial fibrillation in the community: The framingham heart study. JAMA Netw. 2003, 290, 1049-1056. [CrossRef] [PubMed]

20. Wang, S.; Zhang, J.; Lu, W. Sample size calculation for the proportional hazards model with a time-dependent covariate. Comput. Stat. Data Anal. 2014, 74, 217-227. [CrossRef]

21. Barrett-Connor, E.; Cox, D.A.; Song, J.; Mitlak, B.; Mosca, L.; Grady, D. Raloxifene and risk for stroke based on the Framingham stroke risk score. Am. J. Med. 2009, 122, 754-761. [CrossRef]

22. Carroll, K.J. On the use and utility of the Weibull model in the analysis of survival data. J. Control. Clin. Trials 2003, 24, 682-701. [CrossRef]

23. Kannel, W.B.; McGee, D.L.; Castelli, W.P. Latest perspectives on cigarette smoking and cardiovascular disease. J.Card. Rehabil. 1984, 4, 267-277.

24. Antithrombotic Trialists' Collaboration. Collaborative meta-analysis of randomised trials of antiplatelet therapy for prevention of death, myocardial infarction, and stroke in high risk patients. BMJ 2002, 324, 71-86. [CrossRef] [PubMed]

25. Ay, H.; Furie, K.L.; Singhal, A.; Smith, W.S.; Sorensen, A.G.; Koroshetz, W.J. An evidence-based causative classification system for acute ischemic stroke. Am. Neurol. Assoc. 2005, 58, 688-697. [CrossRef] [PubMed]

26. National Health Insurance Service (NHIS). Available online: www.nhis.or.kr/retrieveHomeMain.xx (accessed on 25 June 2020).

27. Lee, J.S.; Park, J.M.; Lee, T.H.; Lee, K.B.; Cho, S.J.; Han, Y.J.; Bae, M.K.; Lee, H.J.; Lee, J.Y. Development of a stroke prediction model for Korean. Korean Neurol. Assoc. 2010, 28, 13-21.

28. American Heart Association. Available online: www.heart.org/en/health-topics/high-blood-pressure/ understanding-blood-pressure-readings (accessed on 25 June 2020).

29. Williams, B.T.; Dixon, R.A. Biochemical testing for acute medical emergencies in four district general hospitals. Br. Med. J. 1979, 1, 1313-1315. [CrossRef] [PubMed]

30. D'Agostino, R.B.; Wolf, P.A.; Belanger, A.J.; Kannel, W.B. Stroke risk profile: Adjustment for antihypertensive medication: The Framingham study. Am. Heart Assoc. 1994, 25, 40-43. [CrossRef] [PubMed]

31. Khosla, A.; Cao, Y.; Lin, C.C.; Chiu, H.K.; Hu, J.; Lee, H. An integrated machine learning approach to stroke prediction. In Proceedings of the 16th ACM SIGKDD International Conference on Knowledge Discovery and Data Mining, Washington, DC, USA, 25-28 July 2010; pp. 183-192.

32. Sundstrom, C. Machine Learning Algorithms for Stroke Diagnostics. Master's Thesis, Biomedical Engineering Chalmers University of Technology-SE-412 96, Gothenburg, Sweden, 2014.

33. Singh, M.S.; Choudhary, P. Stroke prediction using artificial intelligence. In Proceedings of the Industrial Automation and Electromechanical Engineering Conference (IEMECON), Bangkok, Thailand, 16-18 August 2017; pp. 158-161.

34. Alexopoulos, E.; Dounias, G.D.; Vemmos, K. Medical diagnosis of stroke using inductive machine learning. Mach. Learn. Appl. 1999, 20-23.

35. Sut, N.; Celik, Y. Prediction of mortality in stroke patients using multilayer perceptron neural networks. Turk. J. Med. Sci. 2012, 42, 886-893.

36. Agrawal, R.; Imielinski, T.; Swami, A. Mining association rules between sets of items in large databases. In Proceedings of the ACM SIGMOD International Conference on Management of Data, Washington, DC, USA, 25-28 May 1993; pp. 207-216.

37. Quinlan, J.R. Induction of decision trees. Mach. Learn. 1986, 1, 81-106. [CrossRef]

38. Quinlan, J. C4.5: Programs for Machine Learning; Morgan Kaufmann Publishers: San Mateo, CA, USA, 1993.

39. Kim, S.; Oh, S. Decision-tree-based Markov model for phrase break prediction. ETRI J. 2007, $29,527-529$. [CrossRef]

40. Hall, M.; Frank, E.; Holmes, G.; Pfahringer, B.; Reutemann, P.; Witten, L.H. The WEKA data mining software: An update. ACM SIGKDD Explor. Newsl. 2009, 11, 10-18. [CrossRef] 
41. Shi, H.; Wang, H.; Huang, Y.; Zhao, L.; Qin, C.; Liu, C. A hierarchical method based on weighted extreme gradient boosting in ECG heartbeat classification. Comput. Methods Programs Biomed. 2019, 171, 1-10. [CrossRef] [PubMed]

42. Yu, J.; Lee, H.; Im, Y.; Kim, M.S.; Park, D. Real-time classification of Internet application traffic using a hierarchical multi-class SVM. Trans. Internet Inf. Syst. 2010, 4, 859-876. [CrossRef]

43. Yu, J.; Kim, M.; Bang, H.C.; Bae, S.H.; Kim, S.J. IoT as a applications: Cloud-based building management systems for the internet of things. Multimed. Tools Appl. 2016, 75, 14583-14596. [CrossRef]

44. Yu, J.; Lee, B.; Park, D. Real-time cooling load forecasting using a hierarchical multi-class SVDD. Multimed. Tools Appl. 2014, 71, 293-307. [CrossRef]

45. Oh, I.; Lee, J.; Moon, B. Hybrid genetic algorithms for feature selection. IEEE Trans. Pattern Anal. Mach. Intell. 2004, 26, 1424-1437. [PubMed]

46. Hall, M. Correlation-Based Feature Selection for Machine Learning. Ph.D. Thesis, Waikato University, Hamilton, New Zealand, 1998.

47. Yu, L.; Liu, H. Efficient feature selection via analysis of relevance and redundancy. J. Mach. Learn. Res. 2004, $5,1205-1224$.

(C) 2020 by the authors. Licensee MDPI, Basel, Switzerland. This article is an open access article distributed under the terms and conditions of the Creative Commons Attribution (CC BY) license (http://creativecommons.org/licenses/by/4.0/). 